\title{
Target Recognition Algorithm Based on RCS Time Sequence
}

\author{
Xia Tian, Shen Yiying and Hou Chengyu \\ School of Electronics and Information Engineering, Harbin Institute of \\ Technology, Harbin, Heilongjiang Province, 150001, China \\ xiatianhit@126.com
}

\begin{abstract}
Radar Cross Section (RCS) is the information available for nearly all types of radar. $R C S$ is related with lots of factors, therefore the value of RCS of single batch fluctuates greatly, so that fails to extract the space target character. How to use RCS data of space target effectively has the vital significance to improve the target recognition ability of active narrow-band radar. The paper adopts target RCS time series to recognize the target. The existed track data is used to form track information base, radar track matching algorithm based on related functions is presented for the real-time matching of the current dynamic track and the previous track data for the purpose of rapidly finding their RCS time series under the same visual directional angle. A new kind of non-linear and non-stationary time frequency analysis approach Hibert-Huang Transform is then introduced in the paper to decompose the above RCS time series, and then the character index for recognition are extracted from independent intrinsic mode function obtained by the decomposition and the effective target recognition standards are set. The effectiveness and stability of the algorithm presented in the paper are verified by simulated data.
\end{abstract}

Keywords: Target Recognition; RCS Time Sequence; Hibert-Huang Transform; Track Matching

\section{Introduction}

In the military field, monitoring closely the flight conditions of all aircrafts in the native airspace and borders is of great significance to national security. Thus, radar technology emerges as the times requires, in which the reflection of the target to electromagnetic wave (or named re-scattering) is used in detecting, tracking and positioning the target and also in establishing the track information of the target and recognizing the target's type. As is known to all, airplane flies in prescriptive flight segment in flight plan and every flight segment is nearly directional flight. In order to secure the successful accomplishment of some missions in the military field, aircrafts are required to approach the target in specific direction or directional interval to lower radar cross section (RCS) and conceal themselves, so the flight track of the target is traceable. The existing track data are used to construct track information bank. The real-time comparison of current dynamic track and previous track data can recognize rapidly the target type and judge its intention, enhance accuracy in commander's understanding on the situations in the air, giving warning or prompt messages to the target and establishing target reply mechanism in time. According to the target information amount offered, different algorithms can be utilized to calculate the relevance of dynamic track to historical track and match of tracks. In [1], cost-computing approach is adopted in the flight plan management to evaluate and program the quality of tracks. A new selection of factors and weights of cost function and definition of a new indicator for judgment can determine the consistency of tracks. The combination of genetic algorithm with ant colony algorithm can solve the problem of track matching in [2], but its huge amount of 
calculation and complex construction are uneasy for project implementation. Based on these above methods, radar track matching algorithm based on correlation functions is presented in the paper. However, it is not enough to recognize the target by using track comparison alone. During the war, military airplane usually imitates the track of civil plane to conceal itself, aiming at surprise attack. RCS, embodied in radar equation, is the information available for nearly all character-measuring radars and indicates the target's property of scattering. Most of flying targets are complicated ones consisting of multiple independent scattering points. Every independent scattering point of the complicated target would produce an echo signal characterized by amplitude and phase. These echo signals compose a synthetic signal at radar. The variation in the relative position of each scattering object with observation angles or radar frequencies would be followed by the changes in the relative phase of echo signals from each scattering object. As Figure 1(Figure 3.8 in [3]), it shows the relationship of full-size B-26 double-engine (propeller-driven) medium bomber scattering backwards on the wavelength of $10 \mathrm{~cm}$ with visual directional angle. RCS can be seen as a sensitive function adapt to visual directional angle. The accomplishment of target recognition is impossible by using single-time measured value of RCS alone. The longstanding difficult problem is how to use effectively space target RCS to improve target recognition ability of active narrow-band ${ }^{[4]}$. Actually, the time series of target RCS can be utilized to recognize the target. When the ground radar keeps stationary, the target will fly along a definite track and the variations in target track and its attitude are continuous, the function in which target echo intensity fluctuates over time will be formed. According to radar equation, echo intensity sequence can be transformed into RCS sequence. The time series of space target RCS contains abundant target information, so its existing character index can be used to recognize the new target, which is the main content of the study in the paper.

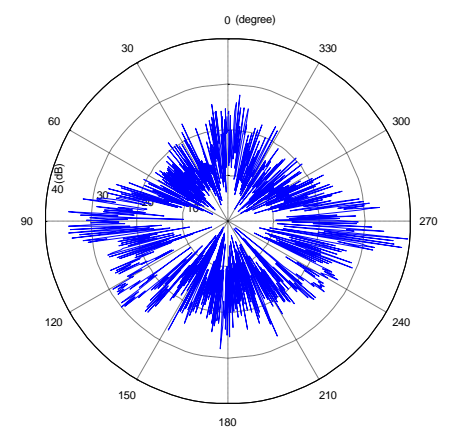

Figure 1. The Diagram Showing the Relationship of Full-Size B-26 Double-Engine (Propeller-Driven) Medium Bomber Scattering Backwards on the Wavelength of $10 \mathrm{~cm}$ with Visual Directional Angle

Two kinds of methods are often applied to extract the character index of RCS time series. One is the conventional statistical analytical method. As in [5], a power spectral density function is adopted to observe RCS time series. In [6], such distribution models as $\chi^{2}$ distribution and lognormal distribution, as well as Kolmogorov method of testing goodness of fit, are used to study the statistical distribution characteristics of the RCS dynamically measured data. However, these algorithms need numerous samples to verify and it is difficult to extract recognition index, and also the effects they obtained is not satisfactory. ARMA model time series analytical method is adopted in [7], RCS time series of the moving space target is known to be non-stationary, so it is very difficult for the conventional time series analytical method to extract its character index and recognize ${ }^{i t^{[8,9]}}$. Thus, non-stationary signal analytical method is used by many scholars nowadays. 
The introduction of Fractional Brownian motion model in [10] is aimed at analyzing the RCS time series and extracting the character. At the present the character extraction and recognition of space target RCS time series present research depends mainly on the application of wavelet transform and fuzzy classification ${ }^{[11,12]}$. However, the application of wavelet analysis is limited by its poor time resolution in the low frequency part and its poor frequency resolution in the high frequency part as well as its reliance on the selection of wavelet function. In order to explore accurate and good properties of time-frequency localization, Norden E. Huang et. al., presented a new algorithm for analyzing the time-frequency of nonlinear, non-stationary signal, i.e., Hilbert-Huang Transform (HHT) in 1996[13], modified in 1999[14]. The essence of HHT is Empirical Model Decomposition (EMD). HHT is proved by Huang et al to have all advantages of the wavelet analysis and more accurate spectral structure, eliminating the resolution fuzziness of the wavelet analysis. Moreover, EMD can get the results with obvious physical meaning directly from spatial domain. For the target recognition in active radar system, the paper uses the existing track data to establish track database and matches the current dynamic track with the previous track data in real time in order to find rapidly their RCS time series under the same visual directional angle and recognize the space target. In this paper, EMD analysis will be conducted on RCS time series to seek the effective method to extract the character.

\section{Track Matching Algorithm Preliminary Option of the Previous Flight Segment and Selection of Matching Points}

It is irrational to use all previous flight routes to correlate with the current dynamic track. The paper makes a space sphere whose center is the current dynamic track point $O$ and radius is range threshold $R_{01}$ at the $\mathrm{t}(\mathrm{t}=1,2,3, \ldots)$ moment and sieves out the flight segment falling into the sphere as the preliminary selection of previous matching flight segment, and then conducts the correlation on the corresponding point, the point of the previous track curve which has the shortest distance with the current dynamic track point $O$.

\subsection{Selection of Correlation Factors}

Through the above processing, the current dynamic track point $O$ matching with the previous track is obtained in the paper. Which one of the previous tracks matches with the dynamic track will be considered in the following from such factors as yawing degree, altitude, velocity and direction.

\subsection{Yawing Factor}

The shortest distance from the current dynamic track point $O$ to the previous track curve $i$ is called yawing distance from this point to the track $i$, which can reflect the possibility in the matching of the track point with its previous flight segment. Thus, yawing distance can be regarded as a factor of correlating the dynamic track with the previous tracks, known as yawing factor. It is defined as:

$Y(t, i) \square 1 / r_{t, i}$

Of which $r_{t, i}$ is the distance from the current dynamic track point $O$ to the corresponding matching point $m_{t, i}$ at the $\mathrm{t}$ moment. It means that yawing factor is inversely proportional to the yawing distance $r_{t, i}$ from the dynamic track point to the flight segment $i$. 


\subsection{Altitude Factor}

The flight altitude is fixed for each flight segment in the flight plan, and every aircraft has the lowest flight altitude [1], so that the flight altitude can be regarded as a factor of correlating the current dynamic track with the previous track, altitude factor. Therefore, altitude factor is in inverse proportion to the absolute value of altitude difference between the current dynamic track point $O$ and the matching point $m_{t, i}$. Shown as the following formula:

$$
H(t, i) \square 1 /\left|h_{t}-h_{m_{t, i}}\right|
$$

\subsection{Velocity Factor}

The velocity of an aircraft is decided by its power system and also dependent on the given environment, task, and space position, so that the flight velocity can be considered as a factor of correlating the dynamic track with the previous tracks, i.e., velocity factor. Therefore, velocity factor is defined to be inversely proportional to the absolute value of the difference between the velocity $v_{t}$ of the current dynamic track point $O$ at the t moment and the velocity $v_{m_{t, i}}$ corresponding to the matching point $m_{t, i}$. Shown as the following formula:

$$
V(t, i) \square 1 /\left|v_{t}-v_{m_{t, i}}\right|
$$

\subsection{Direction Factor}

It is well known that all flights in civil aviation system are in accordance with the flight segment designated in the flight plan and the flights in each flight segment are specific. In order to secure the successful accomplishment of some tasks in the military field, aircrafts are required to approach the target in a definite direction or directional interval for lowering maximally the target RCS and concealing themselves ${ }^{[1]}$. Direction factor can be hence deemed as an important factor of matching the current dynamic track with the previous tracks. Direction factor is accordingly defined to be:

$$
\phi(t, i) \square\left[1-\sin \left(\varphi_{t}-\varphi_{m_{t, i}}\right)\right]
$$

of which $\varphi_{t}$ and $\varphi_{m_{t i}}$ are respectively the motion directions of the current dynamic track points and its matching points.

\subsection{Correlation Index Definition and Judgment Standard}

These factors demonstrate their respective degree of deviation of the current dynamic track point information from the previous track. The assurance of the direction consistency between the dynamic track and the previous flight segment makes direction factor crucial in the track matching algorithm. Apart from direction factor, the other factors can be considered as having the equivalent effect. The correlation index $C(t, i)$ of radar track point $O$ and the flight segment $i$ can be accordingly defined:

$$
C(t, i)=\left[1-\sin \left(\varphi_{t}-\varphi_{m_{t, i}}\right)\right]\left|\frac{F_{Y}}{r_{t, i}}+\frac{F_{V}}{\left|v_{t}-v_{m_{t, i}}\right|}+\frac{F_{H}}{\left|h_{t}-h_{m_{t, t}}\right|}\right|
$$

In the above formula, $F_{Y}, F_{V}, F_{H}$ are weighting coefficients. Considering the actual 
situation, the paper takes one third of the measured standard deviation in the position, velocity and altitude of the reported data, which is the normalization of number within the absolute values. The precise track consistency matching makes each number of the three items inside the absolute value sign exceed one third, so that these three numbers should be taken as the upper bound of one third. The lower bound of the correlation index is set as $T$, the track point which fails to attain $T$ is defined to be unrelated. That is to say, if the following expression is satisfied, they are determined to be correlated and vice versa.

$C(t, i) \geq T$

$K / N$ norm of track correlation can be accordingly used to make the final judgment of track matching, following the increase of the dynamic track points along with time. Fetch natural numbers $N$ and $K$ and make them meet the conditions: $N \geq 2, \quad(N / 2) \leq K \leq N$. Any previous track $i$ will be conducted with correlation detection for $N$ times, of which the expression (6) is assumed to be true for $K$ times and then the dynamic track is determined to be correlated with track $i$.

\subsection{Correlation Monitoring}

In order to monitor effectively the track matching, the track correlation and escape quality are defined. At the $t$ moment, $s_{i}(t)$ indicates the quality of correlating the dynamic track and the previous track $i$. Its calculation is expressed as:

If $C(t, i) \geq T$, there is $\left\{\begin{array}{l}S_{i}(t)=S_{i}(t-1)+1 \\ S_{i}(0)=0\end{array}\right.$

The value of $s_{i}(t)$ represents the times of correlating the dynamic track with the previous track $i$ at the $t$ moment. The correlation quality reflects the accuracy and reliability of correlating two tracks.

The quality $Q_{i}(t)$ of the dynamic track escaping from the previous track $i$ at the $t$ moment is defined as:

When $C(t, i)<T$, there is $\left\{\begin{array}{l}Q_{i}(t)=Q_{i}(t-1)+1 \\ Q_{i}(0)=0\end{array}\right.$

The above expression means that track escape quality can indicate the times of the track $i$ uncorrelated with the dynamic track at the $t$ moment.

In order to enhance the processing speed and simplify the correlation detection, track correlation quality and track escape quality are utilized to monitor the determination of correlation detection in real time. As for the given $K$ and $N$, if the following exists at the $t$ -1 moment:

$Q_{i}(t-1)=N-K+1$

Whatever the subsequent judgment situation may be, the current dynamic track and the track $i$ are inevitably determined to be uncorrelated when $t=N$. Consequently, the detection of correlating the dynamic track with the track $i$ will be terminated at the $\mathrm{t}$ moment. Similarly, if the following exists at $k-1$ moment:

$S_{i}(t-1)=K$ 
The correlation between the dynamic track and the track $i$ would be determined at the $t$ moment.

\section{Target Recognition Algorithm Based on RCS Time Series}

\subsection{Acquisition of RCS time series}

After the matching track corresponding to the dynamic track is obtained, RCS time series of dynamic track on the track segment and the previous track are used to recognize the space target precisely. RCS time series can be derived by reversely deducing the radar equation in use of target point track data and echo energy information from a radar system. The Eq.(11) is the RCS $\sigma$ derived by the monostatic radar propagation equation given by Merrill [15].

$\sigma=\frac{(4 \pi)^{3} P_{r} R^{4}}{P_{t} G_{t} G_{r} \lambda^{2} F_{t}^{2} F_{r}^{2}}$

In the equation, $P_{r}$ and $P_{r}$ are respectively the power of the received signal (the end of the antenna) and the power of the transmitted signal, $G_{r}$ and $G_{t}$ the power gain of the receiving antenna and the power gain of the transmitting antenna separately; $\lambda$ is the wavelength, $F_{t}$ is the propagation factor of the directional diagram from the transmitting antenna to the target, $F_{r}$ is the propagation factor of the directional diagram from the target to the receiving antenna, and $R$ is the distance from the radar to the target. The RCS is calculated point by point by the Eq. (11) to get the RCS time series. The radar equation given by Merrill is known not to be comprehensive for not taking some indefinite radar parameters into account or the influence of weather and system errors, so that there will be errors in some parameters of the radar equation (Eq. (11)). Therefore, a definite error would exist in the RCS time series we got. The errors in the RCS calculation caused by these parameters can be divided into two kinds: one is a fixed value or at most a slowly varying function relative to RCS fluctuation; the other is the high frequency noise of relatively small amplitude. In the following algorithm introduction and experiment, this algorithm will demonstrate such advantage as decreasing the errors in RCS calculation, resulting from inaccurate evaluation.

\subsection{Selection of RCS Time Series for Recognition}

Shown as Figure 2, the angle between the directions of radar observation and airplane flight is recorded as visual directional angle $\theta$. If the difference between the maximum and minimum value of $\theta$ within $1 \mathrm{~min}$ does not exceed $1^{\circ}$, the airplane is determined to in flight-switching condition. The moment at which the fight-switching is finished is the starting point for the effective comparison of RCS time series with intervals. If there is no flight-switching condition in the flight scope, the starting point for effective comparison with intervals is selected as the moment at which the tract starts, and end point is the terminal moment of the shorter one of two comparing tracks. 


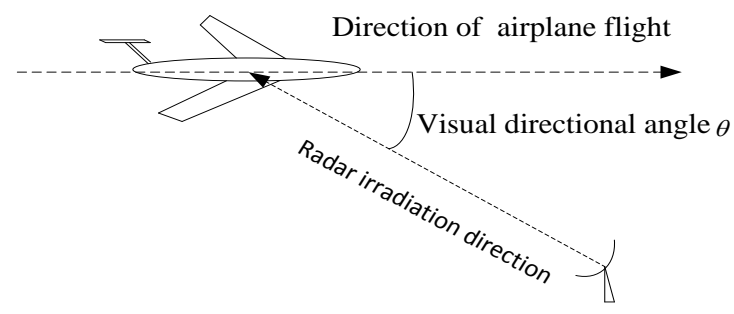

\section{Figure 2. The Diagram of Calculating the Radar Visual Directional Angle}

\subsection{Empirical Mode Decomposition}

Hilbert-Huang Transform (HHT) consists of Empirical Mode Decomposition (EMD) and Hibert Transform. The core of HHT is EMD. In empirical mode decomposition (EMD) algorithm, nonlinear and non-stationary signals can be decomposed into a group of linear and stationary sequence sets, namely, Intrinsic Mode Function (IMF). Every rank of IMF should meet two conditions [13, 14]:

(1) Zero crossings of data and points of extreme value appear alternately, the number of the points is equal or a difference of one between them at most.

(2) The envelope defined by local optimum value at any point requires its mean to be zero.

In the following EMD will be conducted on the time sequence $X(t)$.

Find out all points of extreme value in $X(t)$, and conduct the fitting on the point of maximum value and the point of minimum value respectively with cubic spline function in order to obtain the upper envelope curve $u_{l}(t)$ and the lower envelope curve $l_{l}(t)$ of $X(t)$ and calculate the mean of them which is recorded as $m_{l}(t)$ :

$m_{1}(t)=\frac{u_{1}(t)+l_{1}(t)}{2}$

Subtracting the mean from the initial sequence gets a new sequence removing the low frequency element:

$h_{1}(t)=X(t)-m_{1}(t)$

It is tested whether $h_{1}(t)$ meets the conditions of IMF. If it does, then make $C_{I}(t)=h_{1}(t)$, otherwise make the upper and lower envelope curve of $h_{1}(t)$. Calculate their mean according to the Eq.(12) and record it as $m_{11}(t)$, then compute the following:

$h_{11}(t)=h_{1}(t)-m_{11}(t)$

$h_{l l}(t)$ is tested to meet the conditions of IMF. If it does, then make $W_{l}(t)=h_{l l}(t)$, otherwise repeat (13) and (14) until $h_{l k}(t)$ meets the conditions of IMF and take $W_{l}(t)=$ $h_{l k}(t)$ as the first IMF component of signal $X(t)$. IMF1 component represents the highest frequency element. Subtracting IMF1 from the initial sequence can obtain the remaining sequence $Z_{l}(t)$ removing the high frequency element, namely:

$Z_{1}(t)=X(t)-W_{1}(t)$

Screen $Z_{I}(t)$ again and get the second IMF component $W_{2}(t)$. Repeat above steps until the remaining sequence $Z_{n}(t)$ which cannot be decomposed any more is obtained, or until prearranged numbers of IMF component are got. The sum of each rank of IMF 
component and a mean or a trend item indicates the initial sequence as follows:

$X(t)=\sum_{j=1}^{n} W_{j}(t)+Z_{n}(t)$

\subsection{Space Target Recognition Algorithm Based on EMD}

Shown as (17), any signal can be decomposed into a sum of $n$ intrinsic mode functions (IMF) and one residual item. The number $m_{i}$ of IMF $i$ crossing zero can be calculated, and its normalized instantaneous frequency $F_{i}$ is defined in the paper to be the ratio of its numbers of crossing zero to the length $L$ of its time series, which is expressed as:

$F_{i}=m_{i} / L \quad, i=1,2,3, \cdots, n$

Meanwhile, the energy $e_{i}$ of IMF $i$ is the sum of squares of each point value $w_{i, l}$ on $\mathrm{IMF} i$, which is express as:

$e_{i}=\sum_{l=1}^{L}\left|w_{i, l}\right|^{2} \quad, l=1,2,3, \cdots, L$

$E_{i}$ is supposed to be the percentage of the frequency energy $e_{i}$ on the total sum of each IMF energy, it expresses as follows:

$$
E_{i}=\frac{e_{i}}{\sum_{j=1}^{n} e_{j}} \times 100 \% \quad, i=1,2,3, \cdots, n
$$

The properties of the target RCS frequency can be generally divided into two parts, the rapidly varying part and the slow one. The latter is determined by observation angle and measurement errors, etc, while the former is related to the changes in the target's form, construction and attitude. Taken the actual high-frequency target as an example, the energies of reflecting signals from the nose and the wing of a plane are very different, a tiny variation in the target attitude probably causes a change in irradiation area, making RCS change by dozens decibels. Accordingly, if two RCS time series are the same target, their normalized instantaneous frequencies (defined as $F_{i}$ and $F_{i}^{\prime}$ respectively) followed by their EMD should be greatly similar on the high frequency. Based on this characteristic, the paper sets frequency threshold $D$ as the dividing line between high frequency and low frequency of IMFs. IMFs are arranged in descending order of the instantaneous frequencies and recorded as. If there is:

$F_{j} \geq D \quad, j=1,2,3, \cdots, n$

IMF $j$ will preliminarily selected as the recognition parameter, removing the impact of the errors in the slowly varying relative to RCS fluctuation in RCS calculation (Eq. (11)). Assume that there are $M$ high frequencies meeting the above expression and set the energy threshold $G$ for excluding the impact of high-frequency noises of the smaller amplitude. If there is:

$E_{j} \geq G \quad, j=1,2,3, \cdots, M$

IMF $j$ will be selected as the recognition parameter, otherwise it will be rejected. Suppose if $P$ IMFs meet the requirements and take their instantaneous frequencies as the character frequencies for recognition, then $P$ recognition indexes will be defined as follows: 
$R_{j}=\left|\frac{F_{j}-F_{j^{\prime}}}{F_{j^{\prime}}}\right| \times 100 \% \leq \lambda, \quad, j=1,2,3, \cdots P$.

$\lambda$ is recognition index threshold, which should generally be the positive number less than 0.5. Meet the above expression, there is $S_{i}=1$, otherwise $S_{i}=0$. The total recognition coefficient $S$ is accordingly as follows:

$S=\sum_{i=1}^{K} S_{k}>P / 2$

If $S$ is greater than or equal to a half in the numbers of recognition index, they will be identified to be the same target, otherwise the different targets. If they are identified to be the same target, the system will reset the yawing testing threshold $R_{02}, r_{p, i}<R_{02}$ is used to test the distance of airplane deviating from the expected track. The early warning is given when the distance is greater than preset value, aiming at monitoring the conditions of target flight plan in real time. Figure 3 is the flowchart of the algorithm presented in this paper.

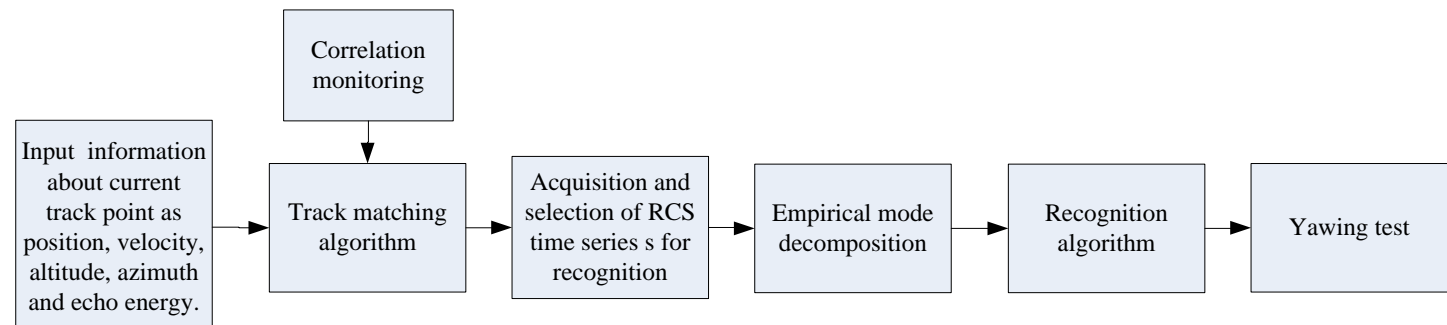

Figure 3. The Flowchart of the Algorithm Presented in the Paper

\section{Simulation Results and Its Analysis}

\subsection{Simulation Database Establishment}

The simulation data is composed of ten previous tracks and two current dynamic tracks obtained by some phased array radar simulation platform. Simulation radar reports the target every 2 seconds and gets back such data as longitude, latitude, altitude, velocity and flight direction of the target at that moment. The RCS value corresponding to each track point of the target is obtained by accessing the RCS values of the following two different types of airplanes. Figure 1 (Figure 3.8 in [3]) demonstrates the relationship of full-size B-26 double-engine (propeller-driven) medium bomber scattering backwards on the wavelength of $10 \mathrm{~cm}$ with visual directional angle. Figure 4 is provided by the data in [16], RCS is measured in the paper in accordance with Boeing 737-type commercial jet model designed in the ratio of $1: 15$ on the conditions of vertical polarization on the frequency of $10 \mathrm{GHz}$. The frequency of measuring the full-size target is one fifteenth of the frequency of $10 \mathrm{GHz}$, i.e., $667 \mathrm{MHz}$. RCS in full size is greater than it in simulation by $23.5 \mathrm{~dB}$. The revised data is shown as Figure 4. The visual directional angle $\theta$ of radar irradiating on the target is reckoned with the azimuth between target flight direction and radar observation (shown as Figure 2). The RCS values corresponding to the visual directional angles are found out in Figure 1 or Figure 4 for evaluation, producing RCS time series. Ten independent previous tracks obtained by the phased array radar simulation platform and two current dynamic tracks and their corresponding RCS time series will not be in full supply, due to the limited pages of the paper. 


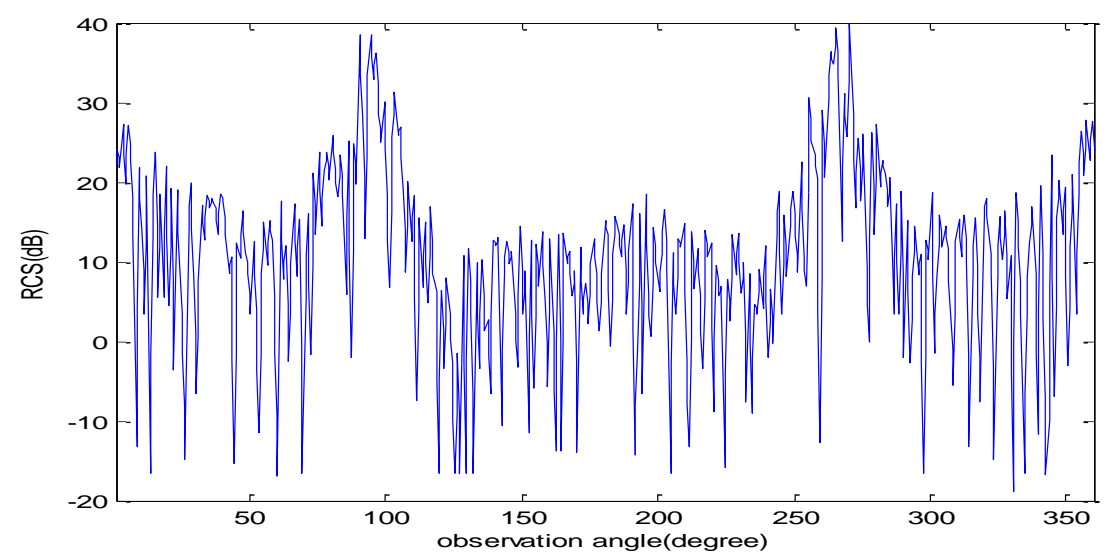

\section{Figure 4. The Relationship between Visual Directional Angle and RCS of Boeing 737-Type Commercial Jet Model on the Condition of Vertical Polarization on the Frequency of $667 \mathrm{MHz}$}

\subsection{Track Matching Results Analysis}

Set range threshold $r_{01}=30 \mathrm{~km}$, weight coefficient $F_{Y}=0.08 \mathrm{~km}, F_{V}=33 \mathrm{~m} / \mathrm{s}, F_{H}=0.01 \mathrm{~km}$, $K=30, N=50$, and set the lower bound value $\mathrm{T}$ of correlation degree as 0.6 , the degree of correlating each points of target track with the previous tracks is reckoned with Eq.(5), the point is determined to be correlation point for exceeding $\mathrm{T}$.

Match two current dynamic target tracks respectively with ten previous tracks of its database and make a judgment, getting the results shown as Table 1 . In the table, 1 represents correlation, and 0 non-correlation.

Table 1. Track Matching Results

\begin{tabular}{lccccc}
\hline & $\begin{array}{c}\text { Previous } \\
\text { track1 }\end{array}$ & $\begin{array}{c}\text { Previous } \\
\text { track2 }\end{array}$ & $\begin{array}{c}\text { Previous } \\
\text { track3 }\end{array}$ & $\begin{array}{c}\text { Previous } \\
\text { track4 }\end{array}$ & $\begin{array}{c}\text { Previous } \\
\text { track5 }\end{array}$ \\
\hline Target track 1 & 1 & 1 & 0 & 0 & 0 \\
Target track 2 & 0 & 0 & 0 & 0 & 0 \\
\hline & $\begin{array}{c}\text { Previous } \\
\text { track6 }\end{array}$ & $\begin{array}{c}\text { Previous } \\
\text { track7 }\end{array}$ & $\begin{array}{c}\text { Previous } \\
\text { track8 }\end{array}$ & $\begin{array}{c}\text { Previous } \\
\text { track9 }\end{array}$ & $\begin{array}{c}\text { Previous } \\
\text { track10 }\end{array}$ \\
\hline Target track 1 & 0 & 0 & 0 & 0 & 0 \\
Target track 2 & 0 & 0 & 0 & 1 & 1 \\
\hline
\end{tabular}

Shown as Table 1 , target track 1 is judged to be or not to be consistent with 10 respective previous tracks, only resulting in correlating with previous track 1 and previous track 2 . It is accordingly determined to match with previous track 1 and previous track 2 . In the same way, target track 2 is determined to match with previous track 9 and previous track 10 .

\section{Target Recognition Results Analysis}

\subsection{Target Recognition Results of Target Track 1 and Previous Track 1}

Shown as Figure 5, the RCS time series corresponding to target track 1 and previous 
track 1 are decomposed with EMD, target track 1 can take the whole sequence as the recognized RCS time series due to no flight-switching condition in flight scope of target track 1 and the greater sequence of previous track 1 than that of target track 1 . So the RCS sequences in accord with target track 1 are only intercepted for EMD. The effective comparing RCS time series is marked in red. The results of decomposing target track 1 with EMD are displayed in Figure 6.
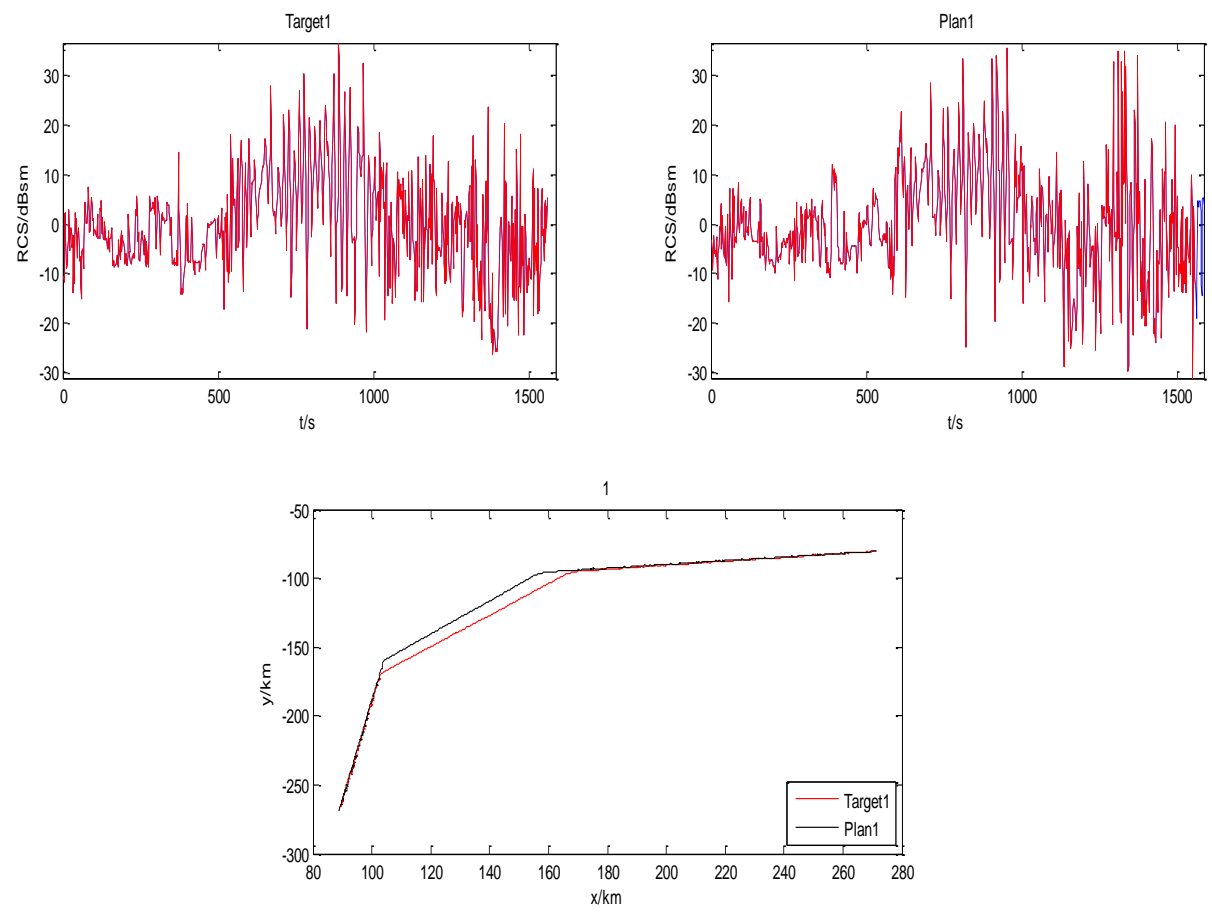

Figure 5 The Corresponding RCS Time Series and Track Matching Results of Target Track 1 and Previous Track 1
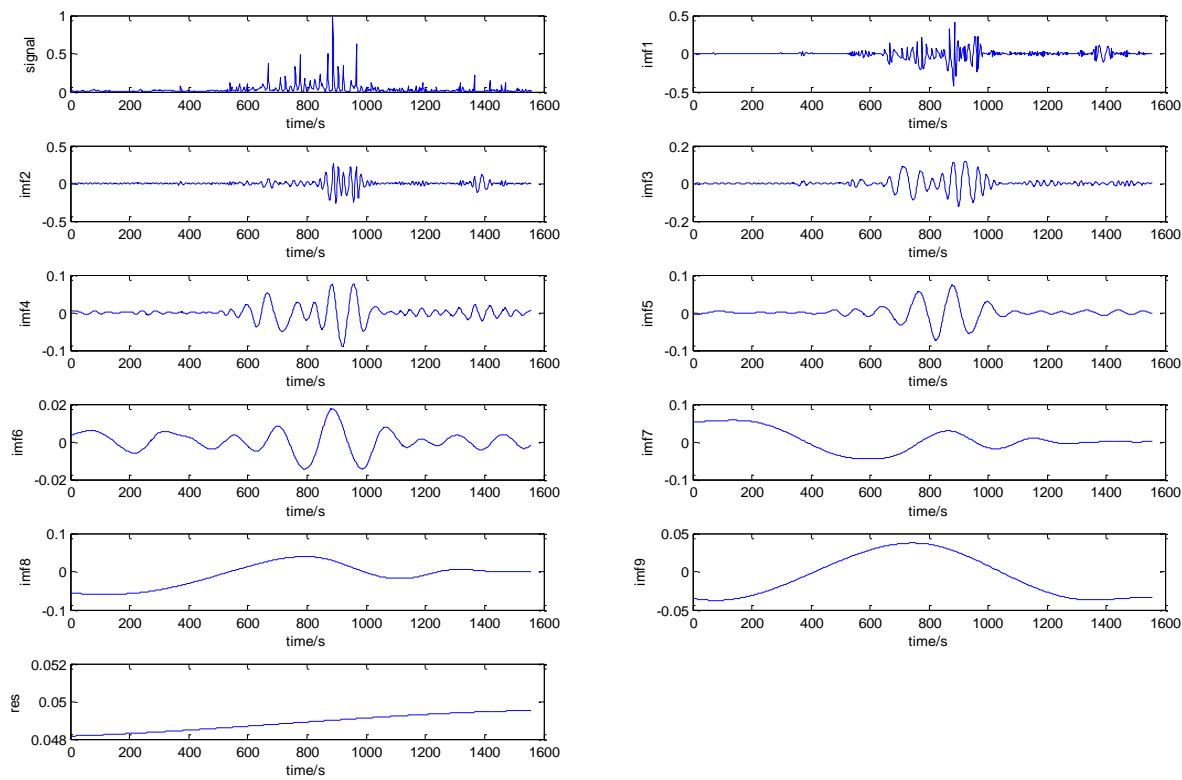

Figure 6. EMD Results of Target Track 1 
The bottom line in Figure 6 shows the envelope information about res, IMFs are arranged in descending order of their normalized instantaneous frequency. The energy percentage and normalized instantaneous frequency of each IMF are reckoned with Eq. (17) and Eq. (19), and previous track 1 can be calculated in the same way, producing the results shown as Table 2.

Chose frequency threshold $D$ to be 0.1 and energy threshold $G 5 \%$, recognition index threshold $\lambda 10 \%$, these above parameters apply to all of data below, so that they will not be presented any more in the following part. Through the computation in accordance with Eq.(20), 3 IMFs of sequence 2 belong to high-frequency IMF and their energies are greater than the energy threshold $G$, which is considered as recognition index, i.e., $\mathrm{P}=3$. According to Eq.(22), relative to the recognition indexes of target track 1 $R_{1}=7.49 \%, R_{2}=3.88 \%, R_{3}=6.30 \%$, the recognition indexes of previous track 1 are less than recognition threshold $\lambda$, so the total recognition coefficient $\mathrm{S}=3$. $\mathrm{S}$ is greater than or equal to one half in the numbers of recognition index $(\mathrm{P} / 2=1.5)$, then they can be determined to be the same target. The obtained data is shown as Table 3.

Table 2. The Normalized Instantaneous Frequency and Energy Percentage of IMFs of Target Track 1 and Previous Track 1

\begin{tabular}{cccccccccc}
\hline IMF & 1 & 2 & 3 & 4 & 5 & 6 & 7 & 8 & 9 \\
\hline F of target 1 & 0.5481 & 0.2978 & 0.1630 & 0.0796 & 0.0411 & 0.0218 & 0.0103 & 0.0077 & 0.0026 \\
F of previous 1 & 0.5071 & 0.2863 & 0.1528 & 0.0886 & 0.0347 & 0.0167 & 0.0077 & .0039 & 0 \\
E of target 1(\%) & 33.28 & 22.53 & 8.26 & 4.40 & 3.72 & 0.34 & 9.21 & 10.16 & 8.11 \\
E of previous 1 & 43.11 & 20.66 & 10.46 & 3.94 & 4.91 & 2.64 & 7.77 & 6.51 & 0 \\
\hline
\end{tabular}

Table 3. Parameters for Recognizing Target Track 1 and Previous Track 1

\begin{tabular}{ccccccc}
\hline & $\mathrm{R} 1$ & $\mathrm{R} 2$ & $\mathrm{R} 3$ & $\mathrm{~S}$ & $\mathrm{P} / 2$ & Conclusion \\
\hline $\begin{array}{c}\text { target track 1 and } \\
\text { previous track 1 }\end{array}$ & $7.49 \%$ & $3.88 \%$ & $6.30 \%$ & 3 & 1.5 & $\begin{array}{c}\text { Same } \\
\text { Target }\end{array}$ \\
\hline
\end{tabular}

\subsection{Target Recognition Results of Target Track 1 and Previous Track 2}

Through the data in the Table 4 and Table 5, we could see that although target track 1 and previous track 2 is correlated. They are determined to be different targets.

Table 4. The normalized instantaneous frequency and energy percentage of IMFs of target track1 and previous track 2

\begin{tabular}{cccccccccc}
\hline IMF & 1 & 2 & 3 & 4 & 5 & 6 & 7 & 8 & 9 \\
\hline F of target 1 & 0.5470 & 0.2973 & 0.1634 & 0.0798 & 0.0412 & 0.0219 & 0.0103 & 0.0077 & 0.0026 \\
F of previous 2 & 0.6100 & 0.3295 & 0.1763 & 0.0888 & 0.0476 & 0.0193 & 0.0142 & 0.0051 & 0.0026 \\
E of target 1(\%) & 33.36 & 22.59 & 8.27 & 4.41 & 3.73 & 0.34 & 9.23 & 8.78 & 9.30 \\
E of previous 2(\%) & 5.35 & 3.67 & 6.84 & 11.95 & 5.22 & 2.94 & 3.90 & 15.28 & 44.86 \\
\hline
\end{tabular}


Table 5. Parameters for Recognizing Target Track 1 and Previous Track 2

\begin{tabular}{cccccc}
\hline & $\mathrm{R} 1$ & $\mathrm{R} 2$ & $\mathrm{~S}$ & $\mathrm{P} / 2$ & Conclusion \\
\hline $\begin{array}{c}\text { target track 1 } \\
\text { and previous } \\
\text { track 2 }\end{array}$ & 10.34 & & & & Different \\
& $\%$ & $7.30 \%$ & 1 & 1 & Targets \\
\hline
\end{tabular}

\subsection{Target Recognition Results of Target Track 2 and Previous Track 9}

Shown as Figure 7, the analysis of sequences after flight switching ending lies in the existence of flight-switching condition for previous track 9. Through the data in the Table 6 and Table 7. We could see that target track 2 and previous track 9 are determined to be different targets.
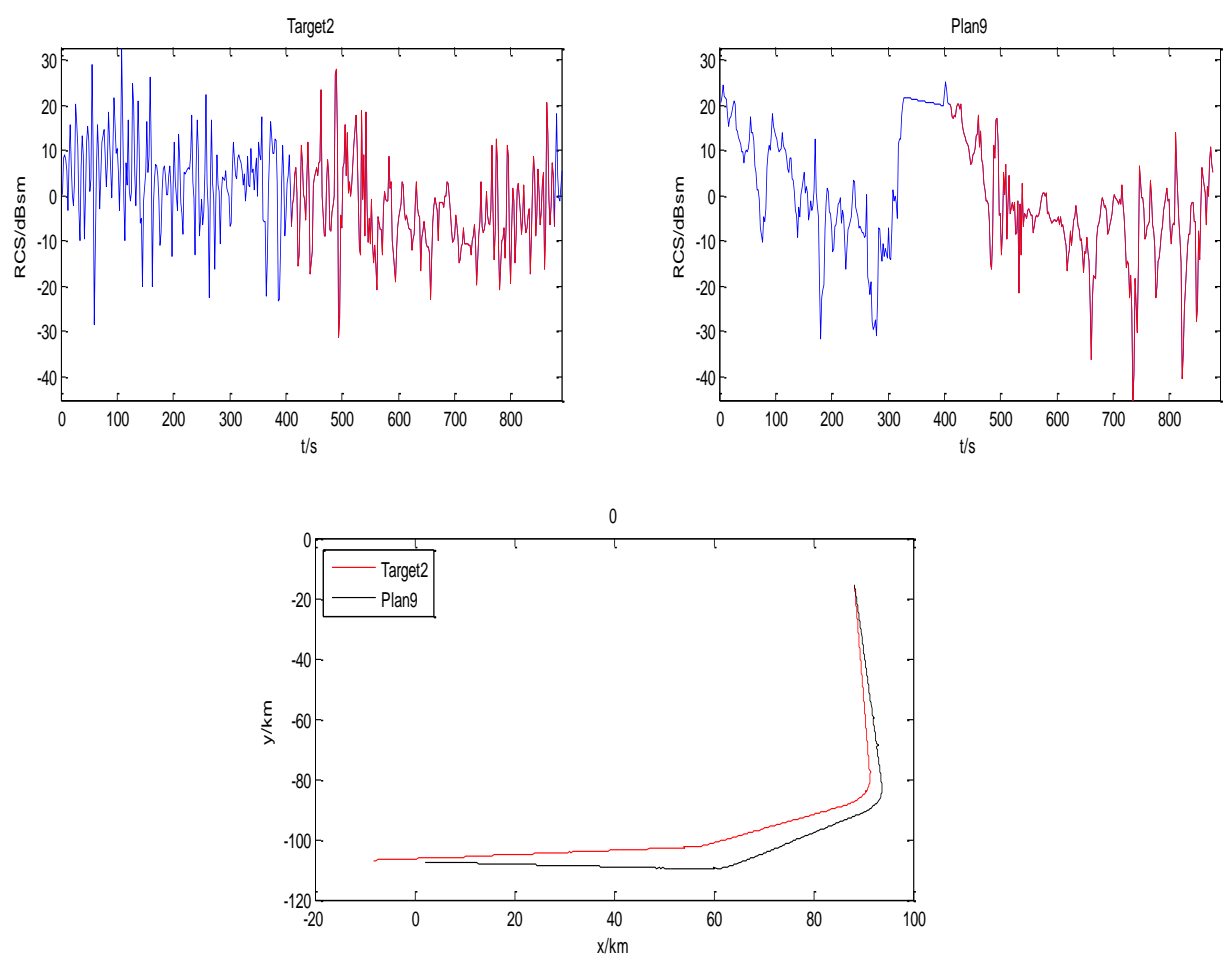

Figure 7. The Corresponding RCS Time Series and Track Matching Results of Target Track 2 and Previous Track 9

Table 6. The normalized Instantaneous Frequency and Energy Percentage of IMFs of Target Track 2 and Previous Track 9

\begin{tabular}{cccccccc}
\hline IMF & 1 & 2 & 3 & 4 & 5 & 6 & 7 \\
\hline F of target 2 & 0.4723 & 0.2681 & 0.1574 & 0.0809 & 0.0383 & 0.0255 & 0.0085 \\
F of previous 9 & 0.6170 & 0.2723 & 0.1277 & 0.0723 & 0.0213 & 0.0085 & 0 \\
E of target 2(\%) & 42.55 & 39.99 & 6.51 & 2.65 & 3.82 & 0.12 & 4.35 \\
E of previous 9(\%) & 7.67 & 14.46 & 26.53 & 6.41 & 6.82 & 38.10 & 0 \\
\hline
\end{tabular}


Table 7. Parameters for Recognizing Target Track 2 and Previous Track 9

\begin{tabular}{ccccccc}
\hline & $\mathrm{R} 1$ & $\mathrm{R} 2$ & $\mathrm{R} 3$ & $\mathrm{~S}$ & $\mathrm{P} / 2$ & Conclusion \\
\hline $\begin{array}{c}\text { target track 2 and } \\
\text { previous track 9 }\end{array}$ & $23.45 \%$ & $1.56 \%$ & $18.92 \%$ & 1 & 1.5 & $\begin{array}{c}\text { Different } \\
\text { Targets }\end{array}$ \\
\hline
\end{tabular}

\subsection{Target Recognition Results of Target Track 2 and Previous Track 10}

In the same way, shown as Table 8 and Table 9, target track 2 and previous track 10 are determined to be the same target. The above simulation results agree with the expected results in simulation plan, having proven the effectiveness of the algorithm.

Table 8. The Normalized Instantaneous Frequency and Energy Percentage of IMFs of Target Track 2 and Previous Track 10

\begin{tabular}{cccccccc}
\hline IMF & 1 & 2 & 3 & 4 & 5 & 6 & 7 \\
\hline F of target 2 & 0.5059 & 0.2813 & 0.1537 & 0.0898 & 0.0426 & 0.0236 & 0.0095 \\
F of previous 10 & 0.4568 & 0.2459 & 0.1442 & 0.0709 & 0.0378 & 0.0165 & 0.0071 \\
E of target 2(\%) & 44.17 & 34.41 & 11.34 & 4.50 & 1.64 & 1.07 & 2.87 \\
E of previous 10(\%) & 47.88 & 14.95 & 9.12 & 8.58 & 4.82 & 7.19 & 7.47 \\
\hline
\end{tabular}

Table 9. Parameters for Recognizing Target Track 2 and Previous Track 10

\begin{tabular}{ccccccc}
\hline & $\mathrm{R} 1$ & $\mathrm{R} 2$ & $\mathrm{R} 3$ & $\mathrm{~S}$ & $\mathrm{P} / 2$ & Conclusion \\
\hline $\begin{array}{c}\text { target track 2 and } \\
\text { previous track 10 }\end{array}$ & $9.71 \%$ & $\begin{array}{c}12.58 \\
\%\end{array}$ & $6.18 \%$ & 2 & 1.5 & $\begin{array}{c}\text { Same } \\
\text { Target }\end{array}$ \\
\hline
\end{tabular}

\section{Conclusions}

The paper uses the existed track data to establish track information bank and matches the current dynamic track with the previous tracks in real time, aiming at finding rapidly target RCS time series under the same visual directional angle for space target recognition. The effectiveness and stability of the algorithm in the paper is proved by simulated data. Moreover, this algorithm can decrease the errors in calculating RCS due to some inaccurate evaluation, which is of great significance to improve the ability of the active narrow-band radar to recognize the target.

\section{References}

[1] L. Fang and C. Yi, "Application of Track Association Algorithm in Flight Plan Management[J]", Information Command Control System \& Simulation Technology, (2005), vol. 27, no. 1, pp. 46-49.

[2] C. Ming and C. Yuhan, "Improved algorithm of associating with radar track and flight plan[J]", Computer Engineering and Applications, vol. 45, no. 10, (2009), pp. 228-230.

[3] Ridenour, L. N., "Radar System Enginerring", MIT Radiation Laboratory Series,vol. New York: McGraw-Hill, (1947).

[4] Nazih N.Y., "Radar Cross Section of Complex Targets", Proceedings of the IEEE, vol. 77, no. 5, (2004), pp. 722-734.

[5] H. Peikang, Y. Hongcheng and X. Xiaojian, "Radar target characteristics", Publishing House of Electronics Indestry, Beijing, (2005).

[6] L. Gang and X. Jiadong, "Study of the Statistical Characterization of Targets", RCS Dynamic Data, Modern Radar, vol. 28, no. 6, (2006), pp. 18-20,39. 
[7] R. Bin, Q. Longhai, X. Shunping and W. Xuesong, "Precession period extraction of ballistic targets based on time series analysis", Chinese Journal of Radio Science, vol. 26, no. 2, (2011), pp.291- 296.

[8] W. Hongyu, "Nonstationary Random Signal Processing and Analysis", National Defence Industry Press, Beijing, (1999).

[9] Z. Xianda and B. Zheng, "Nonstationary Random Signal Processing and Analysis", National Defence Industry Press, Beijing, (1999).

[10] H. Xiaohong, Q. Zhaokun, C. Zengping and Z. Zhenzhong, "The Fractal Feature of Space Object RCS", Chinese Space Science and Technology, vol. 2, no.1, (2005), pp.33-36.

[11] X. Xin, Z. Anjun and J. Shashan, "Recognition Techniques for Space Targets based on RCS", Fire Control \& Command Control, vol. 35, no. 10, (2010), pp.134-136.

[12] .Junguo, Z. Hongzhong and F. Qiang, "Space target recognition algorithm based on RCS sequence", Aerospace Electronic Warfare, vol. 26, no. 6, (2007), pp. 14-16.

[13] Huang N. E., Shen Z. and Long S. R., "The Empirical Mode Decomposition and the Hilbert Spectrum for Nonlinear and Nonstationary Time Series Analysis”, J. ProcR. Soc. Lond.A., (1998).

[14] Huang N. E., Shen Z. and Long S. R, "A New View of Nonlinear Water Waves : the Hilbert Spectrum”, J. Annual Review of Fluid Mechanics, (1999).

[15] M. I. Skolnik, "Radar Handbook,Section ed.", Publishing House of Electronics Indestry, Beijing, (2003).

[16] Howell, N.A., "Design of Pulse Gated Compact Radar Cross Section Range", 1970 IEEE G-AP Int.Prog \& Dig., IEEE Publ.70c 36-AP, (1970) Sept., pp. 187-195.

\section{Authors}

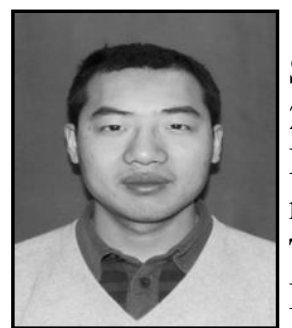

Xia Tian received the B.S. degree in Electronic Information Science and Technology from Harbin Engineering University in 2008 and M.E. degree in Signal and Information Processing from Harbin Institute of Technology in 2010 respectively. From 2010 till now, he has been studying for a Ph.D. in Harbin Institute of Technology. His research area includes Radar Signal and Information Processing.

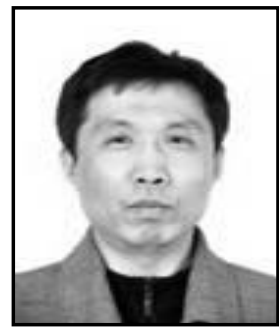

Shen Yiying received B.S, M.S. and Ph.D. degrees in electronic engineering from Harbin Institute of Technology, Harbin, China, in 1982, 1984 and 1999 respectively. Currently, he is a professor at the Department of Electronics and Communication Engineering, Harbin Institute of Technology. His interests mainly include weak-signal detection, radar system and signal processing.

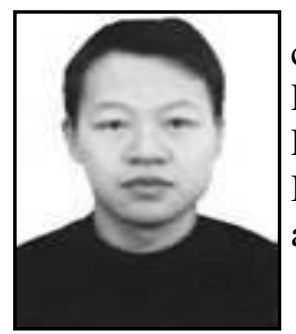

Hou Chengyu received M.S. degree in electronics and communication engineering from Harbin Institute of Technology, Harbin, China, in 2003. Currently, he is a doctoral candidate at the Department of Electronics and Communication Engineering, Harbin Institute of Technology. His research area includes signal processing applied in HF skywave OTH radar. 
International Journal of Signal Processing, Image Processing and Pattern Recognition Vol.8, No.11 (2015) 